\title{
Somatostatin Receptor Imaging May Establish the Neuroendocrine Nature of Unclarified Metastases
}

\author{
Kasper Tholstrup Pedersen ${ }^{1}$, Benedicte Vibjerg Wilson ${ }^{2}$ and Jane Angel Simonsen ${ }^{3}$ \\ ${ }^{1,2,3}$ Odense University Hospital, Sdr. Boulevard 29, 5000 Odense C, Denmark \\ Correspondence should be addressed to: Kasper Tholstrup Pedersen; \\ kasper.tholstrup.pedersen@rsyd.dk
}

Received date: 20 August 2014; Accepted date: 20 October 2014; Published date: 28 December 2015

Academic Editor: Renata Mikołajczak

Copyright (C) 2015. Kasper Tholstrup Pedersen, Benedicte Vibjerg Wilson and Jane Angel Simonsen. Distributed under Creative Commons CC-BY 4.0

\begin{abstract}
Traditionally, functional imaging with somatostatin receptor analogues is used in the primary diagnostics, staging, and monitoring of neuroendocrine tumors (NETs) as well as to assess the potential effect of treatment with (radioactive) somatostatin analogues. We report a case in which a woman was diagnosed with a low grade NET in a liver biopsy, but results from the diagnostic work-up pointed towards a synchronous, high grade, metastasizing malignancy. Because of the patient's poor general health, invasive procedures were deemed unattainable. Somatostatin receptor scintigraphy with the tracer ${ }^{111}$ In-pentetreotide was therefore used as a very specific method of establishing the neuroendocrine nature of all the lesions found, and hence, palliative treatment could be commenced. This case thereby illustrates the feasibility of using somatostatin receptor imaging to establish the neuroendocrine nature of metastases suspected of originating from a synchronous high grade malignancy, when a low grade neuroendocrine tumor has been diagnosed elsewhere and invasive procedures are not possible.
\end{abstract}

Keywords: FDG, somatostatin receptor imaging, NET, unclarified metastases.

\section{Introduction}

Different imaging modalities may be of use in unclarified disease states. Positron emission tomography (PET) with F-18fluorodeoxyglucose (F-18-FDG) depicts cellular glucose metabolism with a high uptake being indicative of a high cellular turnover and, hence, consistent with ongoing infection, inflammation, or high grade malignancy (Hess, et al., 2014). Functional imaging with somatostatin analogues are used for visualizing neuroendocrine tumors
(NETs) expressing somatostatin receptors due to their resemblance with normal neuroendocrine tissue. From time to time, imaging results may be atypical or even conflicting and, therefore, complicating the diagnostic process. Typically, however, different scans complement each other, and the right imaging techniques are useful not only for diagnostic purposes but also for revealing dissemination and guiding biopsy. This case illustrates the use of somatostatin receptor scintigraphy (SRS) with the somatostatin analogue ${ }^{111}$ In pentetreotide 
(Octreoscan $\AA$ ) as a non-invasive means of establishing the neuroendocrine nature of metastases otherwise suspected to arise from a high grade malignancy, when invasive methods were not viable options.

\section{Case}

A 70-year old woman was diagnosed with a $3 \mathrm{~cm}$ inhomogeneous liver lesion, but did not attend a planned biopsy. An ultrasound examination after a year showed no growth of the lesion, and her case was terminated. Eight years later she presented with general discomfort, a $25 \mathrm{~kg}$ weight loss, fever, muscular pain, a raised erythrocyte sedimentation rate, and an elevated plasma concentration of C-reactive protein. A computed tomography (CT) of the chest and abdomen revealed multiple bone metastases throughout the skeleton, an atrophic left liver lobe, dilated hepatic ducts, and some enlarged retroperitoneal lymph nodes. A cholangiocarcinoma was suspected. A subsequent F-18-FDG PET/CT scan of the head, chest, and abdomen (Fig. 1) showed avid FDG uptake in the bone metastases, the known $3 \mathrm{~cm}$ lesion in a now atrophic left liver lobe, the right adrenal gland, the enlarged retroperitoneal lymph nodes, and near the head of the pancreas, supporting the diagnosis of cholangiocarcinoma.
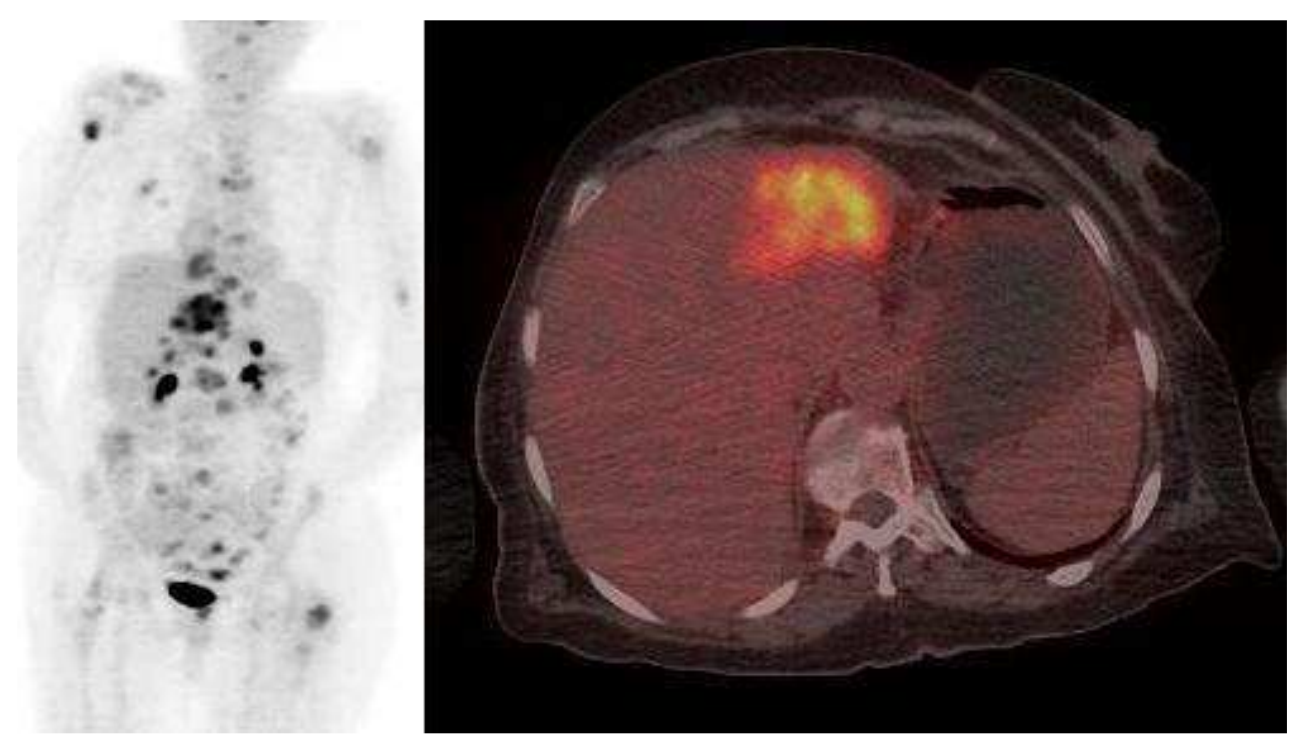

Figure 1: FDG uptake in the bone metastases

An ultrasound-guided biopsy from the liver lesion unexpectedly showed a metastasis from a NET positive in staining for synaptophysin, chromogranin A and CD56, but negative for CK7, CK20, CDX-2, and TTF1 , and with a proliferation index (Ki-67) of $1 \%$. Because of the lack of organ-specific markers, the site of origin could not be determined histologically. A synchronous cancer giving rise to the numerous FDG-avid metastases was still suspected, but further invasive diagnostic procedures were undesirable due to the patient's poor general condition. Because of its high specificity towards neuroendocrine tissue, a SRS with 111In pentetreotide (Fig. 2) was performed showing increased uptake analogous to the FDG scan but in the right adrenal gland, which could not be distinctly separated from the liver. This was interpreted as evidence of the metastases being of neuroendocrine origin despite their avid FDG uptake. Since her general condition had deteriorated significantly, radiation therapy was commenced for pain relief only. She died a few months later. Autopsy was not performed. 

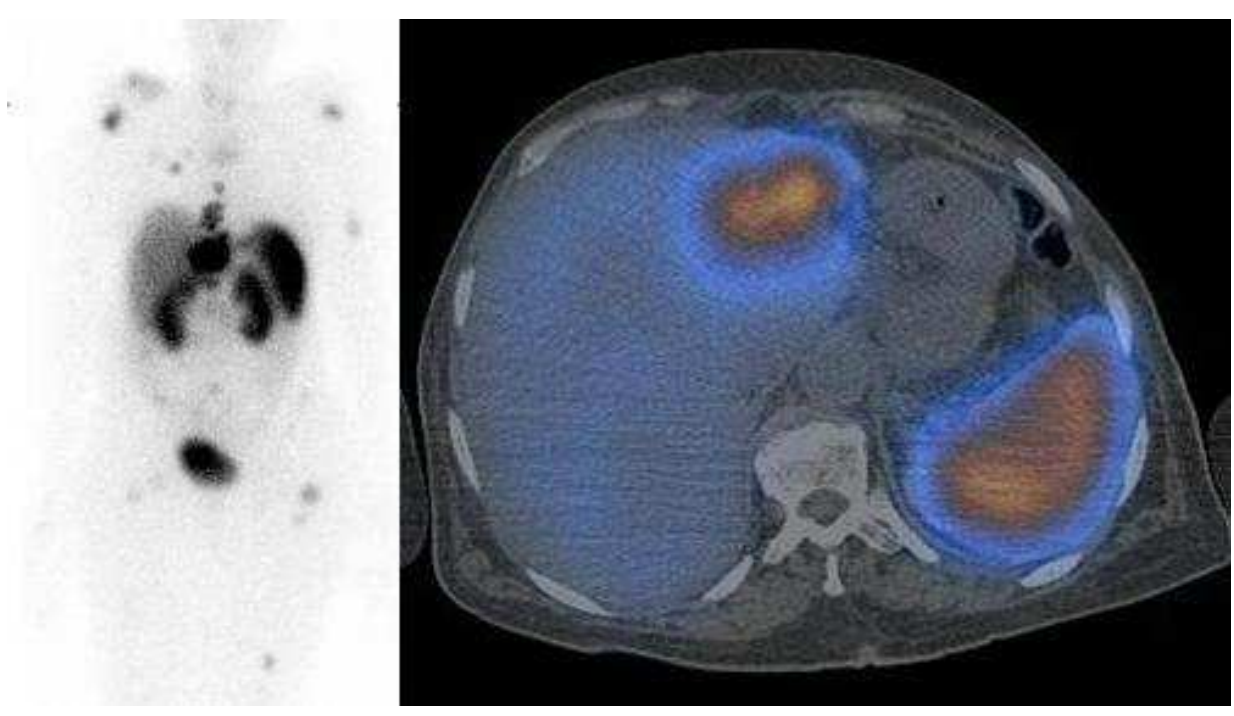

Figure 2: Increased uptake analogous to the FDG scan

\section{Discussion}

NETs originate from cells of the neuroendocrine system. NETs are subdivided into three grades based on their Ki-67 (Bosman, et al., 2010); grade 1, 2, and 3 (G1, G2, and G3) with Ki-67<2\%, 2-20\%, and $>20 \%$, respectively. Typically, the low grade NETs are well differentiated and express somatostatin receptors (SR) in abundance, whereas high grade NETs are often poorly differentiated and seldom express these receptors. SRS has a reported sensitivity of $50-100 \%$ towards identifying primaries and metastases from NETs depending on differentiation and thereby the degree of SR expression, with the lowest sensitivity for poorly differentiated NETs and insulinomas. Poorly differentiated NETs frequently show avid FDG uptake, whereas well differentiated NETs are rarely visualized by FDG PET attributed to their low rate of proliferation (Sundin, Garske and Orlefors, 2007). Furthermore, it has been found that $20-40 \%$ of all the diagnosed NETs were associated with a synchronous neoplasm, typically an adenocarcinoma (Modlin, Lye and Kidd, 2003), which should be kept in mind, if the diagnostic work-up does not "add up".

This case indicates a possible widening of the already established indications of SRS as it may sometimes be used to ascertain the nature of unclarified metastases when a G1
NET has been diagnosed elsewhere and a synchronous cancer is suspected, but invasive procedures are not viable options. Furthermore, a G1 NET may show avid FDG uptake despite its low rate of proliferation, thereby possibly complicating the diagnostic process.

\section{References}

1. Bosman F, Carneiro F, Hruban R, Theise $\mathrm{N}$. "WHO classification of tumours of the digestive system." In: Bosman F, Carneiro F, Hruban R, Theise N, editors. WHO classification of tumours of the digestive system. WHO Press; 2010. p. 417.

2. Hess S, Blomberg BA, Zhu HJ, HøilundCarlsen PF, Alavi A. "The Pivotal Role of FDGPET/CT in Modern Medicine." Acad Radiol. 2014 February 21(2):232-49

3. Modlin IM, Lye KD, Kidd M. "A 5-decade analysis of 13,715 carcinoid tumors." Cancer 2003 February 15;97(4):934-59.

4. Sundin A, Garske U, Orlefors H. "Nuclear imaging of neuroendocrine tumours." Best Pract Res Clin Endocrinol Metab 2007 March 21(1):69-85. 